\title{
A unifying framework of probabilistic reasoning
}

\author{
Rolf Haenni, Jan-Willem Romeijn, Gregory Wheeler \\ and Jon Williamson: Probabilistic logic and probabilistic \\ networks. Dordrecht: Springer, 2011, xiii+155pp, €59.95 HB
}

\section{Jan Sprenger}

Published online: 5 July 2011

(C) The Author(s) 2011. This article is published with open access at Springerlink.com

There is a German proverb that among four lawyers, there are five different opinions. This is, in some sense, also true of probabilistic reasoning: there is a plurality of competing approaches which the outsider finds hard to oversee. Subjective Bayesianism, interval-valued probabilities, Dempster-Shafer theory, classical statistics, the Principle of Maximum Entropy, Evidential Probability and so on. To the untrained eye, they all appear disparate and sometimes idiosyncratic. While it is hard to judge the degree to which they conflict and cohere, it is even harder to come up with a unifying perspective. Perhaps, it is for that reason that relatively few researchers devote their time to investigating parallels, differences and conflicts between these frameworks - among the notable exceptions, there are Teddy Seidenfeld (1979, 1987) and Isaac Levi (2007).

Since these researchers are not any more in the bloom of their youth, it is nice to see that the baton is passed on to the next generation. On not more than 155 pages, Rolf Haenni, Jan-Willem Romeijn, Gregory Wheeler and Jon Williamson make a heroic tour de force through these theories of probabilistic reasoning, with the aim of identifying a unifying overarching framework. The core idea of their book is derived from an analogy with classical first-order logic. There, the central question is the validity of an entailment relation between propositions $\phi_{1}, \ldots, \phi_{N}$ (the premises) and proposition $\psi$ (the conclusion):

$$
\phi_{1}, \ldots, \phi_{N} \vDash \psi \text {. }
$$

Probabilistic logics differ from classical logics in that the valuation function maps these propositions not to the binary values "true" and "false", but to sets $X \subset$ $[0,1]$. The sentence $\phi^{X}$ is standardly interpreted as "the probability of $\phi$ lies in the

\footnotetext{
J. Sprenger $(\bowtie)$

Tilburg Center for Logic and Philosophy of Science, Tilburg University,

P.O. Box 90153, 5000 LE Tilburg, The Netherlands

e-mail: j.sprenger@uvt.nl
} 
set $X$ ". Moreover, instead of verifying or refuting entailment relations such as (1), probabilistic logics focus on determining the set $Y$ for which the entailment relation

$$
\phi_{1}^{X_{1}}, \ldots, \phi_{N}^{X_{N}} \vDash \psi^{Y}
$$

would be satisfied.

Haenni et al. make two main claims that correspond to the two parts of the book. First, they argue that schema (2) provides a general blueprint for theories of probabilistic reasoning. Consequently, they call the search for a proper $Y$ the Fundamental Question of Probabilistic Logic (FQPL), also written $\phi_{1}^{X_{1}}, \ldots, \phi_{N}^{X_{N}} \vDash \psi^{?}$, and they discuss and develop different semantics in which this question can be interpreted.

Second, Haenni et al. argue that credal netwoks provide an efficient means of calculating the set of probabilities $Y$ that satisfy (2). Thus, the first part of the book is more theoretically minded, that is, focused on questions of representation and interpretation, whereas the second part is more application oriented and shows how the theory of credal networks serves as an efficient computational solution to the FQPL.

As the authors happily concede, the book is programmatic in nature. Although it is a research monograph that contains original and stimulating ideas, the main value of the book lies, to my mind, in the way that it bridges the gap between various approaches to probabilistic reasoning and probabilistic argumentation and in showing how the different approaches contribute to the same common goal. The reader gains an effective survey of the various probabilistic logics, their motivations, scope and limits. Furthermore, the book is pleasantly written. Mathematical formalism is employed in a clear and elegant way so that it is not a problem to follow the more technical arguments. Therefore, I do not hesitate to recommend it to the experienced researcher as well as to the aspirant graduate student who wishes to know more about this fascinating area of research.

After introducing the program of the book (Chapter 1) and the standard semantics of probability logic (Chapter 2), the authors pass on to probabilistic argumentation systems (Chapter 3), in particular Dempster-Shafer theory (Dempster 1968; Shafer 1976). Haenni et al. relate this framework to $F Q P L$ and extend the standard semantics of probabilistic logic in order to account for the specifics of probabilistic argumentation systems, in particular, the existence of degree of support and degree of possibility functions. Chapter 4 deals with Kyburg's Evidential Probability theory, whereas Chapter 5 gives an inferential twist to classical statistics. Both frameworks address the question of inductive inference without making use of Bayesian conditionalisation and stay within the realm of observed relative frequencies. This makes it exciting to see how they can be understood as probabilistic logics in the sense of schema (2). Personally, I found it particularly instructive to see how the authors represent Fisher's (1956) notorious fiducial argument in terms of Evidential Probability. Moreover, I very much welcome the inclusion of classical statistical inference which is completely neglected by many logicians. Part I concludes with a discussion of Bayesian statistical inference (Chapter 6) and Objective Bayesianism (Chapter 7) that also provides a fresh look at de Finetti's representation theorem and Carnap's continuum of inductive methods. 
Part II starts out by sketching the theory of credal networks (Chapter 8). This theory, a generalization of the theory of Bayesian networks (Pearl 1988; Neapolitan 1990 ), is a particularly powerful and efficient way of finding a probability interval $Y$ such that FQPL is answered. The focus in this part of the book is on constructing appropriate networks for specific inference problems and on applying this machinery to those problems. Chapters 9-14 return to the approaches discussed in the first part and provide efficient credal network algorithms for computing valid probability models. This is done in a lucidly arranged way although it is not always clear what precisely the reader is supposed to do with the algorithms, once they have been constructed. Perhaps, some applications with real data would have made it easier for the reader to appreciate their value, and to get an idea of where they should be used. However, the authors take time to discuss interesting philosophical questions concerning these algorithms, such as dilation, inference chains and the step-by-step fiducial argument.

The reader may be disappointed by the lack of substantial issues that I have with the book. Partly this is because I applaud the authors' program to systematize and to unify the various ways of probabilistic reasoning, but partly this is because I happened to learn a lot from the book. If I have to identify a weakness, it is the lack of detail in some questions that I would have liked to see discussed in a more elaborate way. For instance, the conflicts between Evidential Probability and Bayesian Conditionalization, the tension between procedural and inferential rationality in classical statistics, and the link between interval probabilities and Bayesian confidence (i.e., credible) intervals would all have deserved a more elaborate treatment. In other words, exchanging the wide scope for more detail and depth at times appears like a favorable trade-off.

However, doing full justice to the topics mentioned elsewhere would simply be an impossible endeavor since each of them is rich enough for a monograph on its own. In that sense, a certain superficiality is a necessary consequence of the programmatic approach that the authors choose. I do not, though, perceive this as a severe drawback. The treatment is extensive enough to give an overview of the crucial open questions, and throughout, an excellent selection of references is provided for the reader who wants to acquire more profound knowledge of a specific topic.

I should also add that nowhere in the book, had I the impression that the four authors succumbed to the problem of the four lawyers mentioned in the outset. By contrast, they have sketched a coherent and convergent picture. True, the area of specialization of each author is duly represented in the book, but this is not a problem: there is no tension between the different chapters, and the authors clearly did their best to present a unified picture and to make the book as coherent and readable as possible. Given that all four of them are researchers with a highly individual profile, this is already a substantive achievement in itself. The reader is rewarded with an oversight that would be hard to gain had a single author written the book.

With a retail price of $€ 59.95$, the book is on upmarket side. Finally, a paperback edition is planned, at the attractive price of $€ 19.95$. If you do not need this book urgently, it may be well worth to wait. 
Open Access This article is distributed under the terms of the Creative Commons Attribution Noncommercial License which permits any noncommercial use, distribution, and reproduction in any medium, provided the original author(s) and source are credited.

\section{References}

Dempster, A.P. 1968. A generalization of Bayesian inference. Journal of the Royal Statistical Society B 30: 205-247.

Fisher, R.A. 1956. Statistical methods and scientific inference. New York: Hafner.

Levi, I. 2007. Probability logic and logical probability. In Probability and inference: Essays in honour of Henry E. Kyburg Jr, ed. W.L. Harper, and G. Wheeler, 255-266. London: College Publications.

Neapolitan, R.E. 1990. Probabilistic reasoning in expert systems. New York: Wiley.

Pearl, J. 1988. Probabilistic reasoning in intelligent systems. San Francisco, CA: Morgan Kaufman.

Seidenfeld, T. 1979. Philosophical problems of statistical inference: Learning from R.A. Fisher. Dordrecht: Reidel.

Seidenfeld, T. 1987. Entropy and uncertainty. In Foundations of statistical inference, ed. I.B. MacNeill, and G.J. Umphrey, 259-287. Dordrecht: Reidel.

Shafer, G. 1976. A mathematical theory of evidence. Princeton: Princeton University Press. 